\title{
Erratum to: Charge transfer in graphene/polymer interfaces for $\mathrm{CO}_{2}$ detection
}

\author{
Kihyeun Kim ${ }^{1, \S}$, Myungwoo Son ${ }^{1, \S}$, Yusin Pak ${ }^{1}$, Sang-Soo Chee ${ }^{1}$, Francis Malar Auxilia ${ }^{1}$, Byung-Kee Lee ${ }^{2}$, \\ Sungeun Lee ${ }^{2}$, Sun Kil Kang ${ }^{2}$, Chaedeok Lee ${ }^{2}$, Jeong Soo Lee ${ }^{2}$, Ki Kang Kim ${ }^{3}$, Yun Hee Jang ${ }^{4}$, Byoung \\ Hun Lee ${ }^{1}$, Gun-Young Jung ${ }^{1}(\varangle)$, and Moon-Ho Ham ${ }^{1}(\varangle)$ \\ ${ }^{1}$ School of Materials Science and Engineering, Research Institute for Solar and Sustainable Energies, Gwangju Institute of Science \& \\ Technology, 123 Cheomdangwagi-ro, Buk-gu, Gwangju 61005, Republic of Korea \\ ${ }^{2}$ Materials \& Devices Advanced Research Institute, LG Electronics, Woomyeon R\&D Campus, 38 Baumoe-ro, Seocho-gu, Seoul 06763, \\ Republic of Korea \\ ${ }^{3}$ Department of Energy and Materials Engineering, Dongguk University, Seoul 04620, Republic of Korea \\ ${ }^{4}$ Energy Systems Engineering, Daegu Gyeongbuk Institute of Science \& Technology, 333, Techno jungang-daero, Hyeonpung-myeon, \\ Dalseong-gun, Daegu 42988, Republic of Korea \\ $\S$ Kihyeun Kim and Myungwoo Son contributed equally to this work. \\ (C) Tsinghua University Press and Springer-Verlag GmbH Germany 2017
}

\section{Erratum to}

Nano Research 2018, 11(7): 3529-3536

https://doi.org/10.1007/s12274-017-1857-z

The order of the authors in the original version of this article was unfortunately incorrect on page 3529 and the first page of the ESM.

Instead of Myungwoo Son ${ }^{1}$, Yusin Pak ${ }^{1}$, Sang-Soo Chee ${ }^{1}$, Francis Malar Auxilia ${ }^{1}$, Kihyeun Kim ${ }^{1}$, Byung-Kee Lee ${ }^{2}$, Sungeun Lee², Sun Kil Kang², Chaedeok Lee², Jeong Soo Lee², Ki Kang Kim³, Yun Hee Jang4, Byoung Hun Lee', $\operatorname{Gun}^{-Y o u n g} \operatorname{Jung}^{1}(\varangle)$, and Moon-Ho $\operatorname{Ham}^{1}(\varangle)$

It should read

Kihyeun Kim ${ }^{1, \$}$, Myungwoo Son ${ }^{1,}$, Yusin Pak ${ }^{1}$, Sang-Soo Chee ${ }^{1}$, Francis Malar Auxilia ${ }^{1}$, Byung-Kee Lee ${ }^{2}$, Sungeun Lee ${ }^{2}$, Sun Kil Kang ${ }^{2}$, Chaedeok Lee², Jeong Soo Lee², Ki Kang Kim³, Yun Hee Jang ${ }^{4}$, Byoung Hun Lee', Gun-Young Jung ${ }^{1}(\varangle)$, and Moon-Ho $\operatorname{Ham}^{1}(\varangle)$

The online version of the original article can be found at https://doi.org/10.1007/s12274-017-1857-z

Address correspondence to Moon-Ho Ham, mhham@gist.ac.kr; Gun-Young Jung, gyjung@gist.ac.kr 